\title{
Uma análise comparativa da precisão de temperaturas e fluxos calculados pelo Método dos Elementos Finitos
}

\author{
Barcelos H. M. ${ }^{* 1}$, Loeffler C. F. ${ }^{2}$ \\ 1 Instituto Nacional de Metrologia, Qualidade e Tecnologia. Duque de Caxias, RJ, Brasil. \\ 2 Programa de Pós-Graduação em Engenharia Mecânica, Universidade Federal do Espírito Santo, Vitória, ES, Brasil. \\ *e-mail: engercules@gmail.com
}

\begin{abstract}
Resumo
Este trabalho é a parte inicial de uma pesquisa mais ampla que objetiva aumentar a qualidade do cálculo das derivadas direcionais em problemas estacionários de campo escalar, resolvidos pelo Método dos Elementos Finitos. Neste método, a determinação do valor das grandezas derivadas tem precisão reduzida, devido à metodologia de cálculo envolver redução da ordem das funções usadas para a aproximação. Mostra-se aqui a base teórica relacionada ao cálculo da variável primal e suas derivadas direcionais, tanto no interior quanto no contorno, e resolvem-se dois problemas bidimensionais, nos quais se demonstra a diferença significativa de precisão dos valores obtidos. Ressalta-se que o procedimento exposto pode ser estendido para casos tridimensionais. Numa próxima etapa da pesquisa, um procedimento alternativo para cálculo do campo de derivadas da variável básica, fundamentado numa associação com o Método dos Elementos de Contorno será desenvolvido.
\end{abstract}

\begin{abstract}
This work is the initial part of broader research that aims to increase the quality of the calculation of directional derivatives in scalar field stationary problems, solved by the Finite Element Method. In this method, the determination of the value of the derivatives has reduced precision, due to the calculation methodology involving reduction of order in the functions used for the approximation. The theoretical basis related to the calculation of the primal variable and its directional derivatives is shown here, both inside and in the boundary, and two two-dimensional problems are solved, in which the significant difference in the accuracy of the obtained values is demonstrated. It should be noted that the exposed procedure can be extended to three-dimensional cases. In the next stage of the research, an alternative procedure for calculating the derivative field of the basic variable will be presented based on the association with the Boundary Element Method.
\end{abstract}

Keywords: Método dos elementos Finitos; Problemas de Campo Escalar; Equação de Laplace.

\section{Introdução}

Grandes desafios estão surgindo com os avanços da engenharia moderna, exigindo a aplicação corrente de métodos numéricos na solução de seus problemas, que permeiam as mais diversas áreas, desde a construção de arrojadas edificações até o projeto de complexos equipamentos mecânicos, passando por aplicações na área do eletromagnetismo, da mecânica da fratura e geofísica. Em contraposição tanto aos procedimentos analíticos de solução, que apresentam enormes restrições matemáticas, quanto às técnicas experimentais, que envolvem elevado custo e dispêndio de tempo (como no caso de protótipos e modelos em escala reduzida), as técnicas numéricas são efetivamente rápidas e flexíveis. Programas sofisticados desenvolvidos em escala comercial permitem alterações ágeis em diversos aspectos: na conformação geométrica dos problemas, nas propriedades constituintes e também nas condições de contorno e iniciais. Nesse contexto, mesmo as formulações relacionadas aos métodos numéricos já consolidados também precisam ser significativamente melhoradas.

O Método dos Elementos Finitos (MEF) é, na atualidade, o mais importante destes métodos baseados na obtenção de soluções aproximadas através de processamento computacional [1]. Fundamenta-se na ideia de discretização do domínio 
em regiões menores, ou seja, grosso modo, na escolha de partes limitadas em meio à infinidade das partículas que compõem o sistema ou volume de controle examinado. Nestas partes, denominadas de elementos finitos, exige-se à obediência de certos requisitos matemáticos importantes, como o equilíbrio, a continuidade e outros tipos de compatibilidade ou restrições [2].

Existem muitos esforços despendidos pela comunidade científica na obtenção de formulações do MEF mais abrangentes, capazes de tornar o método ainda mais atraente. Um exemplo importante consiste do desenvolvimento das técnicas sem malha (meshless). Tais técnicas resolvem os problemas referentes à questão da conectividade, permitindo que os pontos de discretização possam ser distribuídos arbitrariamente e cada região se sobreponha às demais, posicionando-se sem que exista explicitamente uma malha estruturada com elementos. A conectividade pode não ser tão significativa em problemas de pequeno porte; todavia, nos casos tridimensionais, com extensa quantidade de dados, a eliminação da conectividade é um passo importante na redução do trabalho e do custo computacional. Contudo, ainda há outros desafios. A questão da precisão dos resultados das derivadas da variável básica ou primal é um deles.

Uma grande parte dos problemas físicos da engenharia e da física aplicada, quando modelados matematicamente, resulta em equações diferenciais parciais de segunda ordem. Estas envolvem necessariamente uma variável básica ou primal e suas derivadas espaciais. Por exemplo: num problema escalar envolvendo temperatura, os fluxos precisam ser calculados; num problema estrutural, expresso em termos dos deslocamentos, requerem-se os cálculos das tensões. No MEF, a determinação do valor destas grandezas derivadas tem precisão reduzida devido à metodologia de cálculo envolver derivadas das funções usadas para a aproximação da variável primal [3].

Neste trabalho, que é a parte inicial de uma pesquisa mais ampla, mostra-se a base teórica relacionada ao cálculo da variável primal e de suas derivadas direcionais, tanto no interior quanto no contorno, e resolvem-se dois problemas bidimensionais, nos quais se compara a precisão dos valores obtidos. Ressaltase que o procedimento exposto pode ser estendido para casos tridimensionais. Numa próxima etapa da pesquisa, um procedimento alternativo para cálculo do campo de derivadas da variável básica será apresentado.

\section{Equacionamento básico}

São muito numerosos os problemas físicos importantes onde a variável de interesse é uma grandeza escalar $\mathrm{u}\left(\mathrm{x}_{1}, \mathrm{x}_{2}\right)$. Nesta categoria podem ser citadas grandezas como a temperatura, a pressão, a entalpia, a altura piezométrica, o potencial elétrico, a concentração de uma dada substância etc. Na ausência de fontes e variação temporal, se o campo vetorial oriundo do gradiente de $\mathrm{u}\left(\mathrm{x}_{1}, \mathrm{x}_{2}\right)$, apresenta seu divergente nulo em todos os pontos deste domínio, o problema é governado pela Equação de Laplace [1], conforme expresso na Eq. (1), em sua forma diferencial:

$$
\mathrm{k} \nabla^{2} \mathrm{u}\left(\mathrm{x}_{1}, \mathrm{x}_{2}\right)=0
$$

O significado de $\mathrm{u}\left(\mathrm{x}_{1}, \mathrm{x}_{2}\right)$ e $\mathrm{k}$ podem variar, dependendo do problema físico abordado, que compreende um domínio físico finito $\Omega(\mathrm{X})$ e sua fronteira $\Gamma(X)$, onde $X=X\left(x_{1}, x_{2}\right)$ que pode ser dado por um sistema ou por um volume de controle. Nos casos envolvendo transmissão de calor, a Equação de Laplace representa os problemas de difusão ou condução térmica e nos exemplos, apenas por conveniência, o significado de $\mathrm{u}\left(\mathrm{x}_{1}, \mathrm{x}_{2}\right)$ será tomado como temperatura e $\mathrm{k}$ como a constante de condutividade térmica. Finalizando o equacionamento básico, para que um problema linear seja bem-posto, é preciso apenas estabelecer condições de contorno suficientes para o problema, sendo estas essenciais ou naturais. A primeira condição envolve a prescrição da variável básica ou primal u e a segunda a sua derivada normal q [4].

\section{Equações Integrais do MEF}

O MEF é deduzido a partir de uma formulação integral. Tal abordagem parte do princípio que é mais eficaz obter-se uma solução numérica que satisfaz globalmente requisitos como equilíbrio, continuidade etc. em partes do domínio do que efetivamente em cada ponto deste, conforme exigem os métodos ditos diferenciais, como o Método das Diferenças Finitas. Neste último, a equação diferencial de governo deve ser obedecida ponto a ponto, e não de modo mediano ou integral.

Assim, matematicamente, o ponto de partida do MEF pode ser feito, inicialmente, a partir da postulação da denominada forma integral forte associada à Equação de Laplace:

$$
\mathrm{k} \int_{\Omega}\left(\nabla^{2} \mathrm{u}\right) \mathrm{wd} \Omega=0
$$

A função $w\left(x_{1}, x_{2}\right)$ é uma função auxiliar e seu significado depende de cada método. Existem interpretações físicas importantes, que norteiam a dedução dos diferentes métodos numéricos; assim, para o MEF é muito comum se encontrar deduções do método baseada em princípios de energia, como o princípio dos trabalhos virtuais, o princípio da energia 
mínima e outros. Outro princípio importante e muito geral é o dos Resíduos Ponderados [5], que consegue englobar todos os principais métodos numéricos segundo uma interpretação na qual as funções $\mathrm{w}\left(\mathrm{x}_{1}, \mathrm{x}_{2}\right)$ são funções de ponderação, capazes de minimizar os erros na aproximação pela projeção de resíduos num espaço funcional, criado pela escolha de $\mathrm{w}\left(\mathrm{x}_{1}, \mathrm{x}_{2}\right)$ e também pela aproximação admitida para 0 campo $\mathrm{u}\left(\mathrm{x}_{1}, \mathrm{x}_{2}\right)$. Neste trabalho, utiliza-se outro caminho, que parte das propriedades matemáticas que compõem a Teoria das Equações Integrais, que também permite uma dedução simples e geral tanto do MEC e do MEF e de outras técnicas correlatas.

Assim, admitindo certos requisitos para $\mathrm{w}\left(\mathrm{x}_{1}, \mathrm{x}_{2}\right)$ e usando o recurso matemático da integração por partes, pode-se escrever:

$$
\begin{gathered}
\mathrm{k} \int_{\Omega}\left(\nabla^{2} \mathrm{u}\right) \mathrm{wd} \Omega=\mathrm{k} \int_{\Omega} \nabla(\nabla \mathrm{uw}) \mathrm{d} \Omega- \\
\mathrm{k} \int_{\Omega} \nabla \mathrm{u} \nabla \mathrm{wd} \Omega,
\end{gathered}
$$

onde esta é a forma integral fraca associada à Equação de Laplace. Aplicando, então, o Teorema da Divergência no primeiro termo do lado direito da equação anterior:

$$
\mathrm{k} \int_{\Omega} \nabla(\nabla \mathrm{uw}) \mathrm{d} \Omega=\int_{\Gamma}\left(\mathrm{k} \frac{\partial \mathrm{u}}{\partial \mathrm{x}_{\mathrm{j}}} \frac{\partial \mathrm{x}_{\mathrm{j}}}{\partial \mathrm{n}}\right) \mathrm{wd} \Gamma=\int_{\Gamma} \mathrm{qwd} \Gamma
$$

A substituição da Eq. (4) na Eq. (3) resulta em:

$$
-\mathrm{k} \int_{\Omega} \nabla \mathrm{u} \nabla \mathrm{wd} \Omega+\int_{\Gamma} \mathrm{qwd} \Gamma=0
$$

O termo está associado ao fluxo de calor que envolve o contorno e até este ponto não foi feita qualquer aproximação, o que torna a Eq. (5) exata. À luz da teoria de aproximação de funções, percebe-se que as exigências matemáticas de continuidade visando a derivação na função primal $\mathrm{u}\left(\mathrm{x}_{1}, \mathrm{x}_{2}\right)$ se suavizaram, pois que agora nesta se impõe uma derivada de primeira ordem.

Cabe ressaltar que as formulações de muitas técnicas numéricas de solução podem partir tanto da formulação integral forte quanto da forma integral fraca. Sobretudo quando se examinam tais expressões sob o enfoque do Método dos Resíduos Ponderados, é possível estabelecer variantes como: o Método da Colocação, o Método das Subregiões, o Método dos Momentos, o Método de Galerkin e o Método de Mínimos Quadrados, que distinguem todos pela forma com que se escolhe a função auxiliar $w\left(x_{1}, x_{2}\right)$, que toma 0 significado de função de ponderação. Todas estas técnicas, entretanto, se baseiam na aproximação global do domínio $\Omega(\mathrm{X})$ como um todo, o que se traduz em dificuldades operacionais significativas quando se refina o modelo discreto gerado por todas essas técnicas numéricas. No caso do MEF, há uma alteração na filosofia da aproximação: ao invés de se elevar a quantidade de funções ou sua ordem para, então, melhorar a aproximação da solução real em todo o domínio, se procura usar funções mais simples e polinomiais apenas no nível de subdomínios - os elementos finitos - e depois compatibilizá-los entre si através das condições de continuidade e equilíbrio.

\section{Modelo discreto do MEF}

\subsection{Aproximação do campo}

Considera-se o domínio $\Omega(\mathrm{X})$ dividido em subdomínios $\Omega^{\mathrm{e}}(\mathrm{X})$ - os já citados elementos finitos - cujas fronteiras são definidas por pontos nodais. No caso dos elementos triangulares, os pontos nodais se situam nos vértices dos triângulos, conforme mostrado na Fig. (1):

Figura 1 Malha genérica do MEF em duas dimensões.

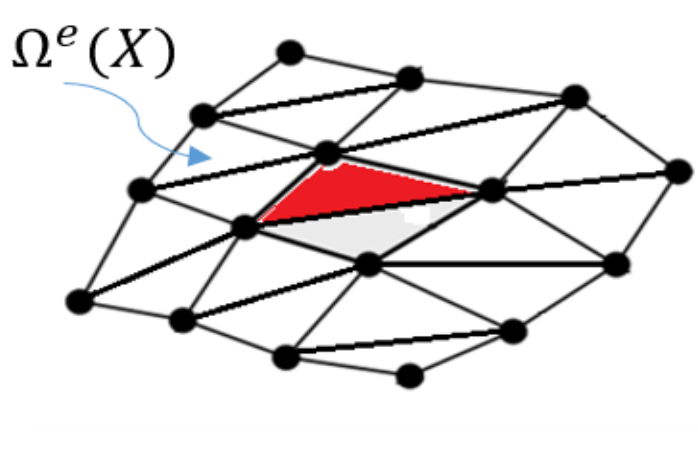

Fonte: Próprio autor.

No MEF, admite-se que os valores de $\mathrm{u}\left(\mathrm{x}_{1}, \mathrm{x}_{2}\right)$ variam espacialmente e de modo aproximado no elemento (vide Fig. (2)), para isto considere a função de interpolação polinomial de ordem 1 na Eq. (6).

$$
\mathrm{u}^{\mathrm{e}}\left(\mathrm{x}_{1}, \mathrm{x}_{2}\right)=\mathrm{a}_{0}+\mathrm{a}_{1} \mathrm{x}_{1}+\mathrm{a}_{2} \mathrm{x}_{2}
$$

Figura 2 - Elemento triangular.

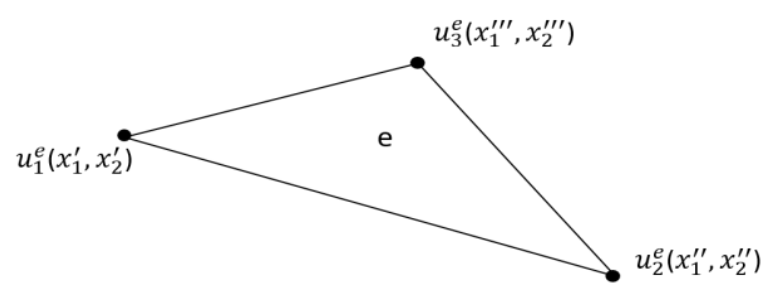

Fonte: Próprio autor.

Sabendo que $u_{1}^{e}, u_{2}^{e}$ e $u_{3}^{e}$ são valores de nodais conhecidos, pode-se escrever:

$$
\boldsymbol{U}^{e}=\left[\begin{array}{l}
u_{1}^{e} \\
u_{2}^{e} \\
u_{3}^{e}
\end{array}\right]=\left[\begin{array}{ccc}
1 & x_{1}^{\prime} & x_{2}^{\prime} \\
1 & x_{1}^{\prime \prime} & x_{2}^{\prime \prime} \\
1 & x_{1}^{\prime \prime \prime} & x_{2}^{\prime \prime \prime}
\end{array}\right]\left[\begin{array}{l}
a_{1} \\
a_{2} \\
a_{3}
\end{array}\right]=\boldsymbol{G} \boldsymbol{A}
$$

$\mathrm{Na}$ Eq. (7) $\mathbf{U}^{\mathbf{e}}$ e A são respectivamente os vetores de potencial e dos coeficientes, enquanto G é a matriz de 
interpolação. Desta maneira os componentes do vetor A podem ser encontrados através da expressão:

$$
\mathbf{A}=\mathbf{G}^{-\mathbf{1}} \mathbf{U}^{\mathbf{e}}
$$

Considerando $\mathbf{G}^{\mathbf{- 1}}=\mathbf{D}$ e os componentes da matriz $\mathbf{D}$ iguais a $D_{i j}$, a função de interpolação $\mathrm{u}^{\mathrm{e}}\left(\mathrm{x}_{1}, \mathrm{x}_{2}\right)$ pode ser reescrita como:

$$
\mathrm{u}^{\mathrm{e}}\left(\mathrm{x}_{1}, \mathrm{x}_{2}\right)=\left[1 ; x_{1} ; x_{2}\right]\left[\begin{array}{ccc}
D_{11} & D_{12} & D_{13} \\
D_{21} & D_{22} & D_{23} \\
D_{31} & D_{32} & D_{33}
\end{array}\right]\left[\begin{array}{c}
u_{1}^{e} \\
u_{2}^{e} \\
u_{3}^{e}
\end{array}\right]
$$

Esta organização possibilita a construção das funções de interpolação $\varphi\left(\mathrm{x}_{1}, \mathrm{x}_{2}\right)$, dadas por:

$$
\left\{\begin{array}{l}
\varphi_{1}\left(x_{1}, x_{2}\right)=D_{11}+D_{21} x_{1}+D_{31} x_{2} \\
\varphi_{2}\left(x_{1}, x_{2}\right)=D_{12}+D_{22} x_{1}+D_{32} x_{2} \\
\varphi_{3}\left(x_{1}, x_{2}\right)=D_{13}+D_{23} x_{1}+D_{33} x_{2}
\end{array}\right.
$$

\subsection{Cálculo da variável primal}

Assim, a função de interpolação $\mathrm{u}^{\mathrm{e}}\left(\mathrm{x}_{1}, \mathrm{x}_{2}\right)$ no elemento pode ser escrita em termos dos potenciais conhecidos nos nós, a saber:

$$
\begin{gathered}
\mathrm{u}^{\mathrm{e}}\left(\mathrm{x}_{1}, \mathrm{x}_{2}\right)=\varphi_{1}\left(x_{1}, x_{2}\right) u_{1}^{e}+\varphi_{2}\left(x_{1}, x_{2}\right) u_{2}^{e}+ \\
\varphi_{3}\left(x_{1}, x_{2}\right) u_{3}^{e}
\end{gathered}
$$

Dentro da formulação clássica do MEF aqui apresentada, o procedimento ostensivamente utilizado é o Método de Galerkin, para o qual as funções de ponderação $\mathrm{w}\left(\mathrm{x}_{1}, \mathrm{x}_{2}\right)$ são escolhidas como sendo as próprias funções de forma. Assim, discretiza-se localmente a Eq. (5) sob um elemento finito $\Omega^{e}(\mathrm{X}) \mathrm{com}$ contorno $\Gamma^{e}(\mathrm{X})$, obtendo-se $\mathrm{n}$ equações do tipo:

$$
\begin{gathered}
\sum_{i=1}^{n} \sum_{j=1}^{n} u_{j} k \int_{\Omega^{e}(X)}\left(\frac{\partial \varphi_{j}}{\partial x_{1}} \frac{\partial \varphi_{i}}{\partial x_{1}}+\frac{\partial \varphi_{j}}{\partial x_{2}} \frac{\partial \varphi_{i}}{\partial x_{2}}\right) d \Omega^{e}(X)= \\
\sum_{i=1}^{n} \int_{\Gamma^{e}(X)}\left(k \frac{\partial u}{\partial x} n_{x}+k \frac{\partial u}{\partial y} n_{y}\right) \varphi_{i} d \Gamma^{e}(X)
\end{gathered}
$$

Da Eq. (12), o termo $u_{j}$ representa os valores nodais de potencial prescrito ou a ser calculado. A conectividade entre os elementos finitos $\Omega^{\mathrm{e}}(\mathrm{X})$ ocorre através nós de interseção (vide Fig. (1)), permitindo-se desenvolver a seguinte equação matricial:

$$
\boldsymbol{K} \boldsymbol{U}=\boldsymbol{F}
$$

A matriz $\mathbf{K}$ é denominada matriz de difusividade global, podendo ser identificada conforme a Eq. (14).

$$
\boldsymbol{K}_{i j}=\int_{\Omega(X)}\left(\frac{\partial \varphi_{j}}{\partial x_{1}} \frac{\partial \varphi_{i}}{\partial x_{1}}+\frac{\partial \varphi_{j}}{\partial x_{2}} \frac{\partial \varphi_{i}}{\partial x_{2}}\right) d \Omega(X)
$$

O vetor global $\mathbf{F}$ representa a interpolação dos fluxos prescritos tanto no contorno do elemento quanto em pontos do domínio, sendo a sua expressão definida pela Eq. (15).

$$
\boldsymbol{F}_{i}=\int_{\Gamma(X)}\left(k \frac{\partial u}{\partial x} n_{x}+k \frac{\partial u}{\partial y} n_{y}\right) \varphi_{i} d \Gamma(X)
$$

A análise do integrando na Eq. (14) mostra que a matriz $\mathbf{K}$ é simétrica devido a propriedade $\mathrm{k}$ ser constante, apresentando desta forma uma característica essencial para uma ágil e eficiente solução computacional do sistema de equações, de onde resultam os valores da variável primal nos nós no vetor $\mathbf{U}$ :

$$
\mathbf{U}^{\mathbf{T}}=\left\{\mathrm{u}_{1}, \mathrm{u}_{2}, \ldots, \mathrm{u}_{\mathrm{n}}\right\}
$$

\subsection{Cálculo do fluxo no interior}

A construção da matriz de interpolação $\mathbf{D}$ é dada por:

$$
\begin{aligned}
& \mathbf{D}= \\
& \frac{1}{\operatorname{det}[G]}\left[\begin{array}{ccc}
x_{1}^{\prime \prime} x_{2}^{\prime \prime \prime}-x_{1}^{\prime \prime \prime} x_{2}^{\prime \prime} & x_{1}^{\prime \prime \prime} x_{2}^{\prime}-x_{1}^{\prime} x_{2}^{\prime \prime \prime} & x_{1}^{\prime} x_{2}^{\prime \prime}-x_{1}^{\prime \prime} x_{2}^{\prime} \\
x_{2}^{\prime \prime}-x_{2}^{\prime \prime \prime} & x_{2}^{\prime \prime \prime}-x_{2}^{\prime} & x_{2}^{\prime}-x_{2}^{\prime \prime} \\
x_{1}^{\prime \prime \prime}-x_{1}^{\prime \prime} & x_{1}^{\prime}-x_{1}^{\prime \prime \prime} & x_{1}^{\prime \prime}-x_{1}^{\prime}
\end{array}\right],
\end{aligned}
$$

Sendo o determinante de G calculado conforme a Eq. (18):

$$
\begin{gathered}
\operatorname{det}[G]=\left(x_{1}^{\prime \prime} x_{2}^{\prime \prime \prime}-x_{1}^{\prime \prime \prime} x_{2}^{\prime \prime}\right)+\left(x_{1}^{\prime} x_{2}^{\prime \prime}-x_{1}^{\prime \prime} x_{2}^{\prime}\right) \\
+\left(x_{1}^{\prime \prime \prime} x_{2}^{\prime}-x_{1}^{\prime} x_{2}^{\prime \prime \prime}\right)
\end{gathered}
$$

Pode-se demonstrar que o determinante da matriz G corresponde a duas vezes a área $A_{t}$, do elemento [6], ou seja :

$$
\operatorname{det}[G]=2 A_{t}
$$

Em problemas de campo escalar, como os descritos pela Equação de Laplace, a determinação das derivadas da variável primal, denominados aqui genericamente por fluxos, são tão importantes quanto a determinação de $\mathrm{u}\left(\mathrm{x}_{1}, \mathrm{x}_{2}\right)$. O cálculo destes fluxos $\mathrm{q}^{\mathrm{e}}$ no interior de cada elemento é dado pelo gradiente da função de interpolação $\mathrm{u}^{\mathrm{e}}\left(\mathrm{x}_{1}, \mathrm{x}_{2}\right)$, considerando a expressão:

$$
\mathrm{q}^{\mathrm{e}}\left(\mathrm{x}_{1}, \mathrm{x}_{2}\right)=-\mathrm{k} \nabla \mathrm{u}^{\mathrm{e}}\left(\mathrm{x}_{1}, \mathrm{x}_{2}\right)
$$

Matricialmente, a Eq. (.20) escreve-se como:

$$
\left[\begin{array}{l}
q_{1}^{e} \\
q_{2}^{e}
\end{array}\right]=-\mathrm{k}\left[\begin{array}{lll}
\frac{\partial \varphi_{1}}{\partial x_{1}} & \frac{\partial \varphi_{2}}{\partial x_{1}} & \frac{\partial \varphi_{3}}{\partial x_{1}} \\
\frac{\partial \varphi_{1}}{\partial x_{2}} & \frac{\partial \varphi_{2}}{\partial x_{2}} & \frac{\partial \varphi_{3}}{\partial x_{2}}
\end{array}\right]\left[\begin{array}{l}
u_{1}^{e} \\
u_{2}^{e} \\
u_{3}^{e}
\end{array}\right]
$$

E desta maneira tem-se que:

$$
\left[\begin{array}{l}
q_{1}^{e} \\
q_{2}^{e}
\end{array}\right]=-\mathrm{k}\left[\begin{array}{lll}
D_{21} & D_{22} & D_{23} \\
D_{31} & D_{32} & D_{33}
\end{array}\right]\left[\begin{array}{l}
u_{1}^{e} \\
u_{2}^{e} \\
u_{3}^{e}
\end{array}\right]
$$


O fluxo $\mathrm{q}^{\mathrm{e}}$ pode ser considerado como o uma aproximação do fluxo no centroide do elemento "e" (vide Fig. (3)), sendo a sua precisão condicionada ao refinamento de malha.

Figura 3 - Posicionamento do fluxo no elemento "e".

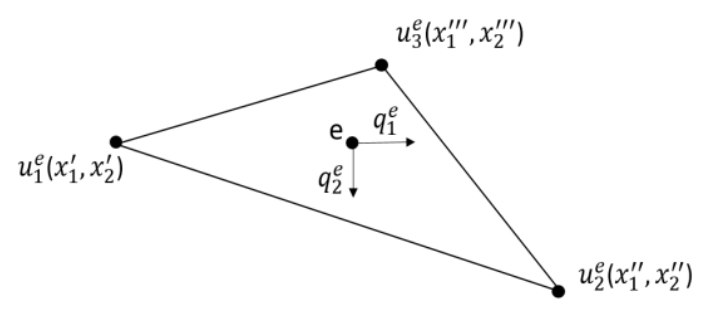

Fonte: Próprio autor.

Está claro que o cálculo dos fluxos envolve derivadas da função de forma. Conseguintemente, sua aproximação numérica é maior, acarretando problemas de precisão, que precisam ser minimizados: seja através do refinamento da malha, ou seja, aumentando a quantidade de elementos finitos ou escolhendo elementos cuja interpolação seja de mais alta ordem. Aqui, neste trabalho, interessa somente avaliar a extensão desta aproximação.

\subsection{Cálculo do fluxo no contorno}

Existem formulações mistas do MEF que, tal como faz - Método dos Elementos de Contorno, resolve diretamente valores dos fluxos incógnitos na fronteira do sistema. Contudo, isto não ocorre na formulação clássica. Os valores dos fluxos no contorno são aproximados por aqueles calculados no centroide dos elementos que tangenciam o contorno através de suas arestas. Consideram-se como fluxos internos aqueles que estão localizados no centroide dos elementos que não tangenciam o contorno. A Fig. (4) ilustrativa o exposto:

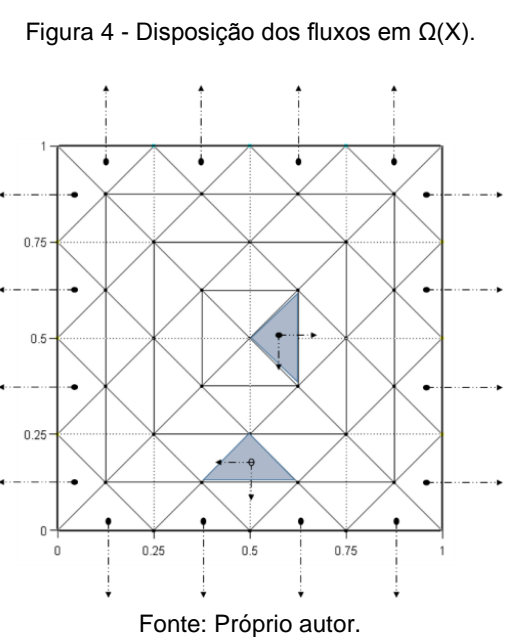

O cálculo dos fluxos no contorno e no interior não apresentaria grandes diferenças não fosse o cuidado que se deve tomar com relação à discretização nos cantos, pois uma quina é o ponto de encontro de contornos com diferentes normais. Assim, certas técnicas mais elaboradas cuidam do cálculo destas grandezas quando são abordados problemas mais complicados e com conformação geométrica mais elaborada. O fato é que a precisão dos valores da variável primal e sua derivada no contorno é efetivamente diferente. Os exemplos que se seguem demonstram essa distinção na qualidade dos resultados.

\section{Simulações numéricas}

Nos dois exemplos que se seguem foram testadas cinco malhas com níveis de refinamento distintos, de modo a se verificar a convergência dos resultados com 0 aumento da quantidade de pontos nodais. O erro médio relativo emr\% (vide Eq. (23)) é dado em valores percentuais e calculado comparando os valores numéricos obtidos com os valores analíticos, que estão disponíveis nos dois exemplos resolvidos, onde o termo $V_{\text {analit }}$ representa o maior valor analítico obtido.

$$
\mathrm{emr} \%=\frac{100}{n} \sum_{i=1}^{n}\left|\frac{v_{i}^{\text {num }}-v_{i}^{\text {analit }}}{V_{\text {analit }}}\right|
$$

Os problemas escolhidos apresentam um campo relativamente elaborado, expresso por funções harmônicas e exponenciais, ou seja, não polinomiais. Por clareza, a Fig. (5) mostra duas destas malhas, empregadas na solução dos exemplos, as malhas 1 e 4. Os dados precisos das cinco malhas são mostrados na Tab. (1).

Figura 5 - Malha 1 à esquerda e Malha 4 à direita.

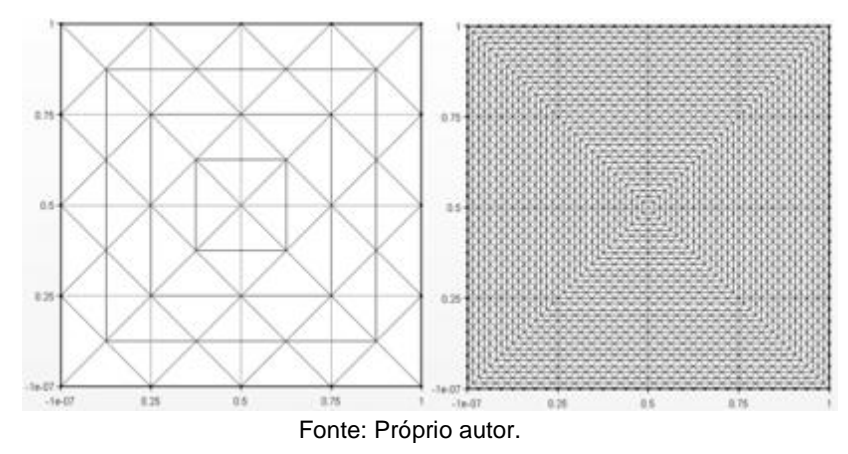

Tabela 1: Característica das malhas.

\begin{tabular}{ccc}
\hline Malhas & № total de nós & $\begin{array}{c}\text { № de } \\
\text { elementos }\end{array}$ \\
\hline 1 & 41 & 64 \\
\hline 2 & 145 & 256 \\
\hline 3 & 545 & 1024 \\
\hline 4 & 2113 & 4096 \\
\hline 5 & 8321 & 16384 \\
\hline
\end{tabular}




\subsection{Primeiro exemplo}

Um domínio quadrado é submetido a um campo harmônico de temperatura $\mathrm{u}\left(\mathrm{x}_{1}, \mathrm{x}_{2}\right)$, ou seja, obediente à Equação de Laplace, na forma [7]:

$$
u\left(x_{1}, x_{2}\right)=\cosh \left(x_{1}\right) \operatorname{sen}\left(x_{2}\right)
$$

As condições de contorno são aplicadas seguindo a Eq. (24) de modo que nas arestas horizontais o valor da temperatura é conhecido e nas arestas verticais o fluxo é prescrito. A Fig. (06) mostra a conformação geométrica e as condições de contorno do exemplo em apreço.

Figura 6 - Domínio para o primeiro exemplo.

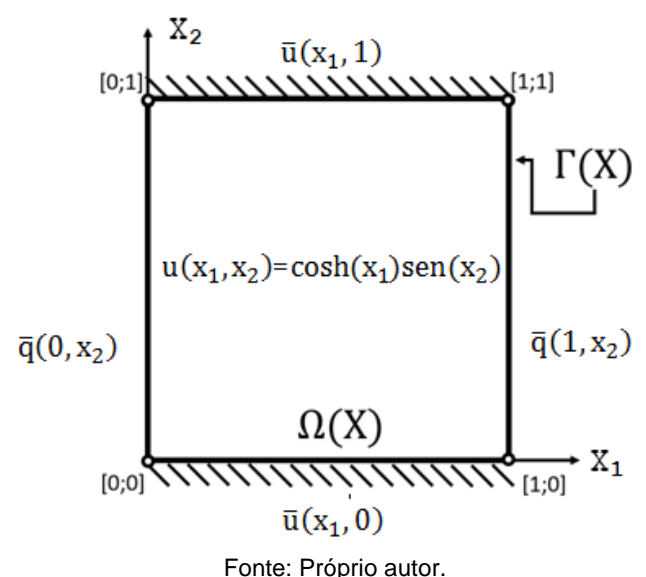

Inicialmente, são apresentados os resultados da temperatura em todos os pontos nodais onde esta grandeza foi calculada. Nota-se claramente uma redução acentuada do erro global com o refinamento, conforme apresentado na Fig. (07). O menor erro global foi de $0,00568 \%$.

Figura 7 - Resultado para o cálculo da variável primal no 1ํe exemplo.

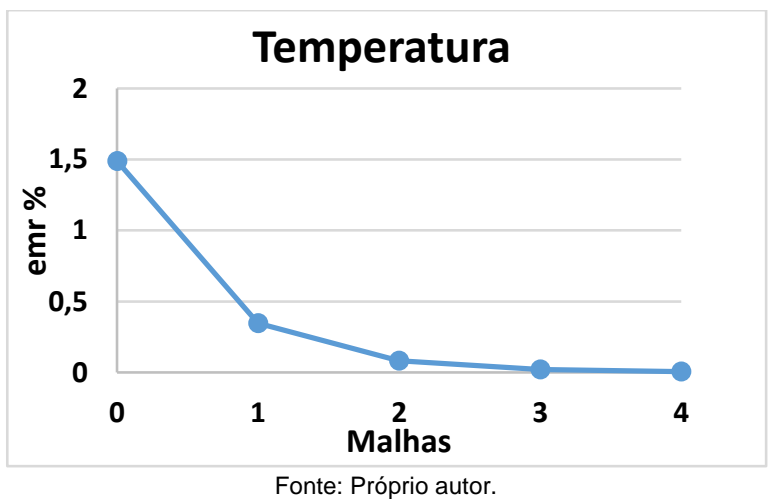

A distribuição dos fluxos internos pode ser observada na Fig. (08). Conforme esperado, os valores do erro nos fluxos são maiores, devido à maior aproximação gerada pela derivação das funções de forma. Para a malha mais refinada, o menor erro no cálculo do fluxo no contorno (vide Fig. (09)) é de 0,425875\%, ainda maior do que o menor valor médio dos fluxos no interior $(0,134516 \%)$, apresentados na Fig. (10).

Figura 8 - Campo de fluxo interno para o $1^{\circ}$ exemplo.

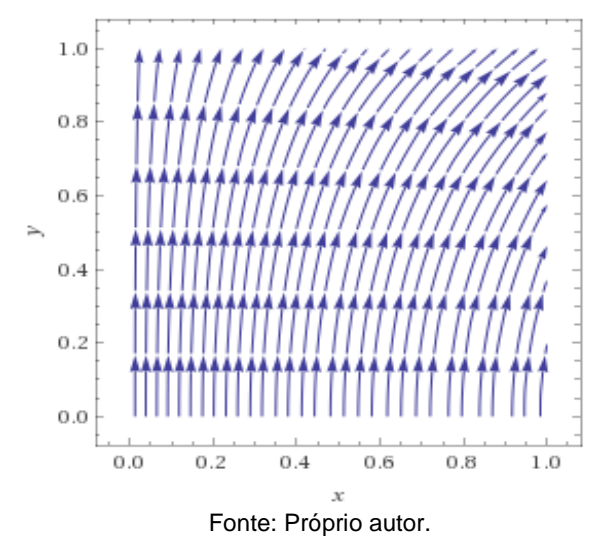

Figura 9 - Resultado para o cálculo da derivada da variável primal no contorno para o $1^{\circ}$ exemplo.

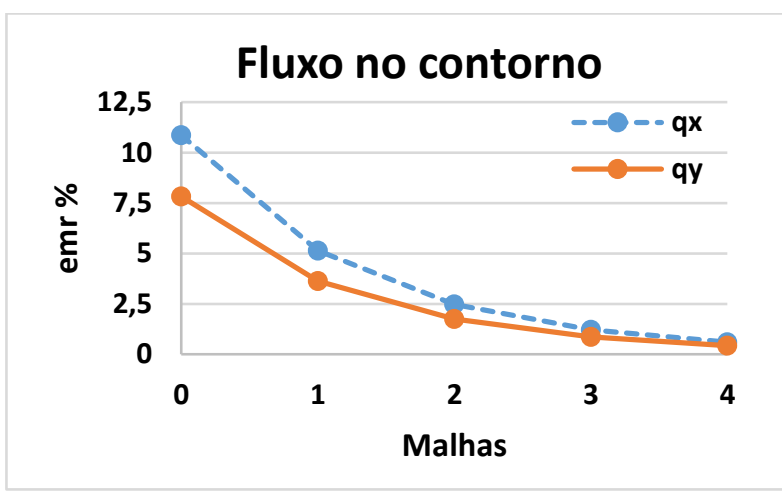

Fonte: Próprio autor.

Uma razão se deve ao fato de que dois dos pontos nodais usados para se calcular os fluxos estão efetivamente sobre o contorno, mas o terceiro não. Uma possibilidade, portanto, seria a contabilização de uma média usando apenas os nós situados sobre o contorno.

Figura 10 - Resultado para o cálculo da derivada da variável primal no interior para o $1^{\circ}$ exemplo.

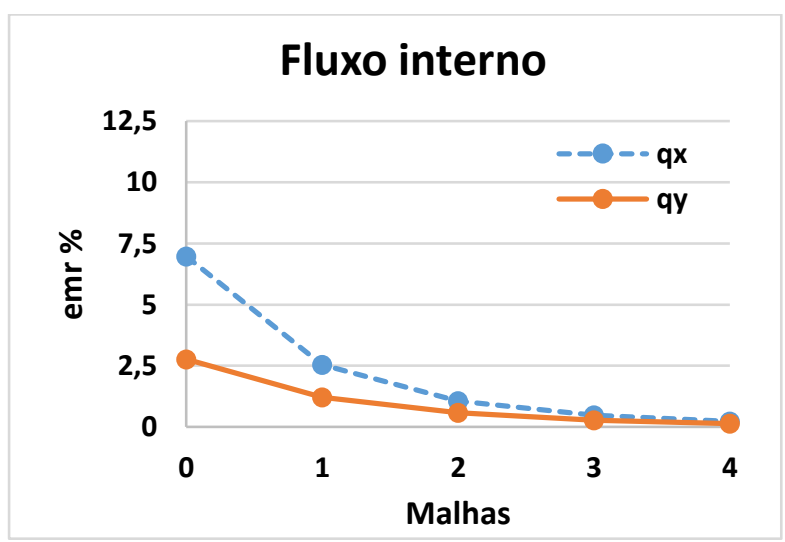

Fonte: Próprio autor. 


\subsection{Segundo exemplo}

Um domínio igualmente quadrado também é submetido a um campo harmônico de temperatura $\mathrm{u}\left(\mathrm{x}_{1}, \mathrm{x}_{2}\right)$, agora dado pela expressão:

$$
u\left(x_{1}, x_{2}\right)=1+\frac{\operatorname{senh}\left(\frac{\pi x_{2}}{6}\right)}{\operatorname{senh}(\pi)} \operatorname{sen}\left(\frac{\pi x_{1}}{6}\right)
$$

Este campo de temperaturas é ainda mais elaborado que o anterior [8]. As condições de contorno aplicadas são similares, de forma a se poder fazer uma comparação mais equânime: nas arestas horizontais o valor da temperatura é imposto e nas arestas verticais o fluxo é prescrito. A Fig. (11) mostra as características deste exemplo.

Figura 11 - Domínio para o segundo exemplo.

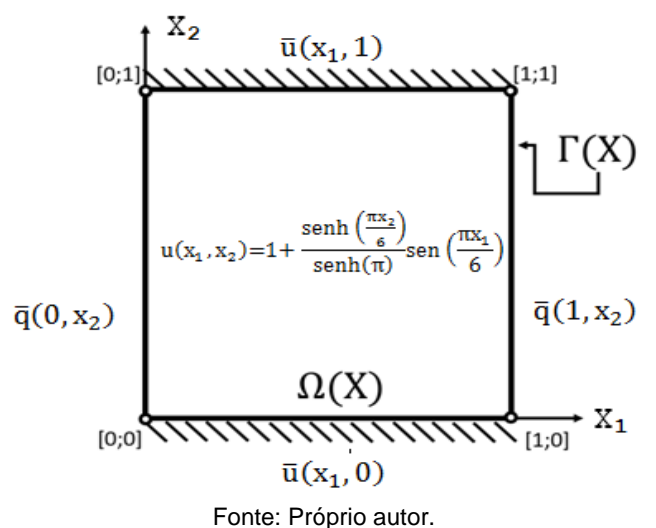

No gráfico da Fig. (12) observa-se, que igualmente ao exemplo anterior, o erro na temperatura foi menor para as malhas mais refinadas. Para melhor avaliação, o menor valor de erro obtido foi de 0,024215\%. O comportamento de tal curva de convergência denota certa exaustão na capacidade do modelo aqui empregado para o MEF. Para melhorar os resultados, é necessário usar elementos finitos com interpolação de ordem mais alta.

Figura 12 - Resultado para o cálculo da variável primal no $2^{\circ}$ exemplo.

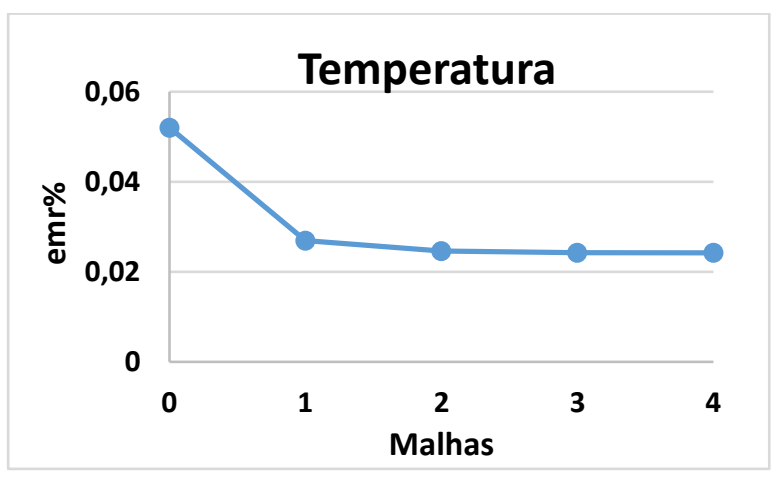

Fonte: Próprio autor.

Para compreender como o ocorre a distribuição do campo vetorial em $\Omega(\mathrm{X})$ observe a Fig. (13). Neste problema a curva de erro se reduziu gradativamente conforme a Fig. (14) e Fig. (15). Mais uma vez, o erro nos fluxos do contorno foi maior do que o erro cometido no cálculo dos fluxos internos. O menor erro no interior, obviamente para a malha mais refinada, foi de $0,160995 \%$ enquanto no contorno foi de $0,684650 \%$. Estes valores são maiores do que os obtidos no exemplo anterior, atestando a maior complexidade deste exemplo.

\section{Figura 13 - Campo de fluxo interno para o $2^{\circ}$ exemplo.}

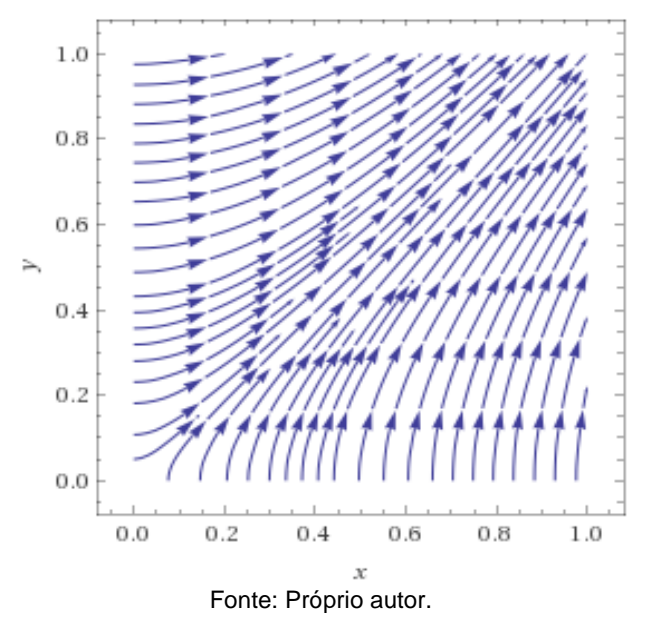

Figura 14 - Resultado para o cálculo da derivada da variável primal no contorno para o $2^{\circ}$ exemplo.

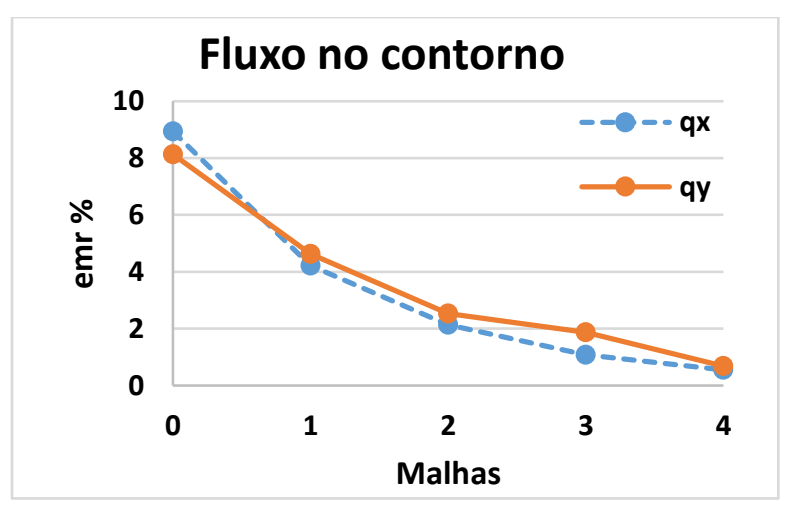

Fonte: Próprio autor.

Figura 15 - Resultado para o cálculo da derivada da variável primal no interior para o $2^{\circ}$ exemplo.

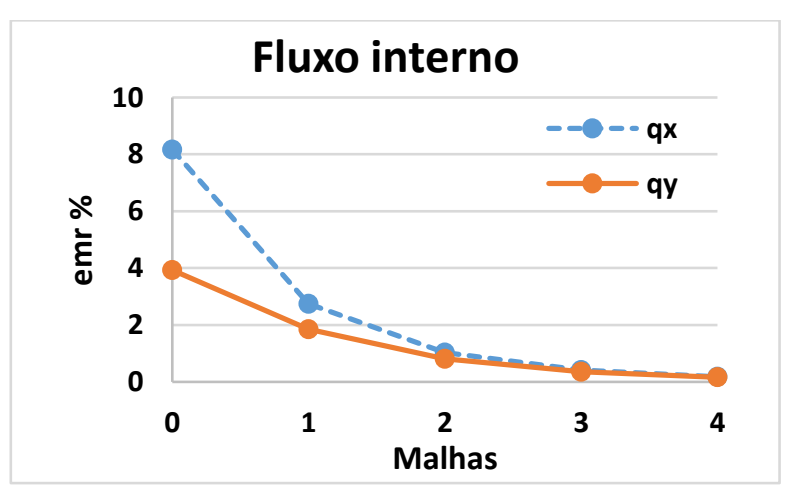

Fonte: Próprio autor. 


\section{Discussão dos resultados}

Os dois exemplos aqui apresentados confirmam a expectativa teórica de que a precisão do MEF no cálculo dos fluxos, seja no interior ou no contorno, é significativamente inferior à dos valores da variável primal ou temperatura.

Sem partir para a adoção de elementos de ordem superior, que aumentariam a qualidade numérica da solução, mas manteriam a diferença na qualidade dos valores de fluxo e da temperatura, outros procedimentos podem ser implementados para melhor aproximar o cálculo dos fluxos e dando continuidade aos propósitos desta pesquisa.

Uma primeira abordagem seria usar funções clássicas de base radial (FBR) [9] para interpolar o campo de temperaturas e depois derivar este campo para obtenção dos fluxos. O emprego das FBR juntos aos métodos numéricos tem sido bem-sucedido e é crescente. Tais funções são de fácil operacionalização e possuem respaldo matemático sob a ótica da teoria moderna da aproximação, sobretudo quando se trata de aproximar dados esparsos em várias dimensões [9]. Sua simplicidade é evidente: são funções cujo valor depende exclusivamente da distância radial entre um ponto base e um ponto campo e nunca são negativos.

Embora seja feita uma derivação do campo primal da função de interpolação, um conjunto de valores nodais - não apenas aqueles pertinentes a um elemento podem entrar no cômputo. Porém, pode-se fazer uma interpolação global, envolvendo todos os pontos nodais, usando FBR com suporte pleno, ou restringindo arbitrariamente o conjunto de pontos nodais, através de FBR com suporte compacto.

Outra possibilidade, mais interessante, consiste do acoplamento do MEF com o Método dos Elementos de Contorno (MEC), num pós-processamento. Os valores das variáveis primais obtidos externamente com o MEF são utilizados na equação integral do MEC para cálculo das derivadas direcionais internas. A equação integral do MEC não realiza derivações na aproximação do campo e é correlata a uma sentença de resíduos ponderados, relacionada ao cálculo dos fluxos [5]. Por essa razão, apresenta uma precisão numérica superior ao esquema clássico do MEF.

\section{Referências}

[1] REDDY, J. N. An Introduction to The Finite Element Method. Texas: McGraw-Hill, 2005.

[2] BATHE, K. J. Finite Element Procedures. New Jersey: Prentice-Hall, 1995.
[3] ZIENKIEWCZ, O; TAYLOR, R.; ZHU, J.Z. The Finite Element Method: Its Basis and Fundamentals. Oxford: Butterworth-Heinemann, 2013.

[4] BREBBiA, C. A.; TELlES, J. C. F.; WROBEL, L.C. Boundary element techniques. Berlin: Springer Verlag, 1984.

[5] BREBBIA, C. A.; WALKER, S. Boundary element techniques in engineering. London: NewnesButterworths, 1980.

[6] SOUZA, R.M. O Método dos Elementos Finitos Aplicado ao Problema de Condução de Calor. Belém: Universidade Federal do Pará, 2003.

[7] INGBER, M. S.; RUDOLPHI T.J. Solution of potential problems using combinations of the regular and derivative boundary integral equations. Appl. Math. Modelling, 14, 536-543. 1990.

[8] TELLES J. C. F; PRADO A. A. (1993): Hypersingular formulation for 2-D potential problems, in Aliabadi M.H. and Brebbia C.A. (Eds.). Advanced Formulations in Boundary Element Methods. Amsterdam: Elsevier, cap. 6,1993.

[9] BUHMANN, M.D. Radial basis function: Theory and implementations. London: Cambridge University Press, 2003. 